\title{
Tea, coffee and oral cancer risk
}

\author{
Abstracted from \\ Galeone C, Tavani A, Pelucchi C, et al. \\ Coffee and tea intake and risk of head and neck cancer: \\ pooled analysis in the international head and neck cancer epidemiology consortium. \\ Cancer Epidemiol Biomarkers Prev 2010; 19: 1723-1736. \\ Address for correspondence: Mia Hashibe, Division of Public Health, \\ Department of Family and Preventive Medicine, University of Utah School of Medicine, \\ 375 Chipeta Way, Suite A, Salt Lake City, UT 84108, USA. E-mail: mia.hashibe@utah.edu
}

\section{Question: Is there a relationship between coffee and tea intake and head and neck cancers?}

Data sources Pooled individual-level data from nine case-control studies of head and neck cancers, including 5,139 cases and 9,028 controls.

Study selection Nine case-control studies were selected from the International Head and Neck Cancer Epidemiology (INHANCE) consortium pool of 33 studies, which included information on coffee (caffeinated and decaffeinated) and tea drinking and cancer of the oral cavity and pharynx. Seven studies also included information on laryngeal cancer.

Data extraction and synthesis Data from individual studies were checked for inconsistencies and pooled in a standardised way into a common database, including a range of sociodemographic, behavioural, lifestyle and health information. Data on consumption across studies were then converted into cups of de/caffeinated tea or coffee per day. The association between head and neck cancers and caffeinated coffee, decaffeinated coffee or tea intake was assessed by estimating the odds ratios (OR) and the corresponding $95 \%$ confidence intervals $(95 \% \mathrm{Cl})$ using a two-stage random-effects logistic regression model with the maximum likelihood estimator. Pooled ORs were also estimated with a fixed-effects logistic regression model. In addition, a test for heterogeneity among studies was conducted.

Results Caffeinated coffee intake was inversely associated with the risk of cancer of the oral cavity and pharynx: the ORs were 0.96 (95\% Cl, 0.94-0.98) for an increment of one cup per day and 0.61 ( $95 \% \mathrm{Cl}, 0.47-0.80)$ in drinkers of $>4$ cups per day versus nondrinkers. This latter estimate was consistent for different anatomic sites (OR, $0.46 ; 95 \% \mathrm{Cl}, 0.30-0.71$ for oral cavity; OR, 0.58; $95 \%$ $\mathrm{Cl}, 0.41-0.82$ for oropharynx/hypopharynx; and OR, 0.61; 95\% $\mathrm{Cl}, 0.37-1.01$ for oral cavity/pharynx not otherwise specified) and across strata of selected covariates. No association of caffeinated coffee drinking was found with laryngeal cancer (OR, 0.96; 95\% $\mathrm{Cl}, 0.64-1.45$ in drinkers of $>4$ cups per day versus non-drinkers). Data on decaffeinated coffee were too sparse for detailed analysis, but indicated no increased risk. Tea intake was not associated with head and neck cancer risk (OR, 0.99; 95\% Cl, 0.89-1.11 for drinkers versus non-drinkers).

Conclusions This pooled analysis of case-control studies supports the hypothesis of an inverse association between caffeinated coffee drinking and risk of cancer of the oral cavity and pharynx.

\section{Commentary}

Tea and coffee are the most commonly consumed hot beverages worldwide. Numerous studies have investigated the association between their consumption and risk of oral and pharyngeal cancers. However, results from these studies were inconsistent due to a number of factors including small study size, lack of focus on coffee or tea and poor control for confounders such as smoking and alcohol drinking; the prime risk factors for these cancers. Given the uncertainty of the association, this review is very relevant. It also serves as a prime example of how meta-analysis works by pooling data from small studies to harness adequate statistical power for the detection of relatively weak associations.

The methodology of the review can be described as rigorous and well thought out. In five studies, controls included patients who were hospitalised for acute non-neoplastic conditions, not related to tobacco smoking and alcohol drinking. Population-based controls were used in the remaining four studies. Data collection was consistent and face-toface interviews were conducted in all studies. Furthermore, the authors assessed the comparability of collected data and validated the collection instrument by examining blank questionnaires from all individual studies and assessing the wording of the interview questions among studies. Questions about caffeinated coffee, decaffeinated coffee and tea drinking were similar across studies, although the exact wording differed.

Four of the nine included studies were conducted in Europe, while four were conducted in North America and one in Central America. The majority of cases were men, $76 \%$ of oral cavity and pharyngeal cancers and $90 \%$ of laryngeal cancers. Cases over 50 years of age were the largest group, $32 \%$ of cancers of the oral cavity and pharynx and $28 \%$ of laryngeal cancers. Furthermore, most cases were non-Hispanic whites, $86 \%$ of oral and pharyngeal cancers and $95 \%$ of laryngeal cancers. Not surprisingly, cases were less educated than controls, more often smokers, and heavy alcohol drinkers.

Results showed that caffeinated coffee was inversely related with the risk of cancer of the oral cavity and pharynx, especially with an intake of more than 4 cups per day. The protective effect was similar across oral cavity and pharyngeal sites. No association of caffeinated coffee drinking was found with cancer of the larynx. The risk estimates of cancer of the oral cavity and pharynx for caffeinated coffee drinking were heterogeneous between studies. Sources of heterogeneity included different patterns of alcohol drinking and tobacco smoking in various populations. Caffeinated coffee drinking was moderately correlated with tobacco $(\mathrm{r}=0.24, \mathrm{P}<0.001)$ and alcohol $(\mathrm{r}=0.14, \mathrm{P}<0.001)$ consumption. Nonetheless, adjustment for alcohol drinking and tobacco smoking did not materially modify 
any of the risk estimates and showed that the inverse association between caffeinated coffee and cancer of the oral cavity and pharynx was similar in the strata of tobacco smoking and alcohol drinking. Moreover, adjustment for body weight and for vegetable and fruit consumption did not change the risk estimates.

A sensitivity analysis was conducted and summary ORs were calculated after the exclusion of one study at a time. This analysis did not reveal any notable change in the estimates, showing that results were not driven by any single study, with ORs for cancer of the oral cavity and pharynx varying between 0.58 and 0.68 . The reported inverse association between caffeinated coffee intake and cancer of the oral cavity and pharynx was consistent across strata of potential confounders and effect modifiers, and in both European and American populations. However, information on decaffeinated coffee indicated a lack of association with risk of cancer of the oral cavity/pharynx and larynx. This could be due to limited data availability as both the frequency of consumption and the amount of decaffeinated coffee consumed were low.

The review had a number of limitations. These included a lack of data on duration of coffee drinking, preparation methods and other exposure factors such as the strength of consumed coffee. Furthermore, the chemical composition of coffee beverages varies according to the source plant used, eg Arabica or Robusta. In general, recall bias is inherent to case-control studies; however, in this review, it is unlikely that the recall of coffee drinking was different on the basis of disease status, as coffee is not commonly known to affect risk of oral cavity and pharyngeal cancers.

In addition to evidence from case-control studies, the protective effect of coffee intake against cancers of the upper digestive and respiratory tracts has been suggested in prospective cohort studies. The Miyagi Cohort Study in Japan showed that the risk of oral, pharyngeal and oesophageal cancers was inversely associated with coffee consumption. ${ }^{1}$ Similarly, Ren and colleagues reported that coffee was inversely associated with oesophageal adenocarcinoma during some follow-up periods. However, the same study noted a borderline significant association between coffee consumption and gastric cardia cancer. ${ }^{2}$ Moreover, coffee drinking has been inversely related to other cancers such as colorectal ${ }^{3,4}$ and endometrial cancers. ${ }^{5}$

The scientific literature has shown that the effects of coffee drinking extend beyond cancer prevention, with conflicting evidence on its impact on the cardiovascular system, especially between men and women. A study from Japan suggested that coffee may have favourable effects on mortality due to cardiovascular disease, especially coronary heart disease, in women. ${ }^{6}$ Giggey and colleagues found that greater coffee intake in men was associated with steeper age-related increases in systolic blood pressure and pulse pressure, particularly beyond 70 years of age and in overweight to obese men. The same study did not find significant relations of coffee intake to blood pressure or pulse pressure in women. ${ }^{7}$ Furthermore, consumption of caffeine can affect various traditional cardiovascular risk factors, including a slight increase in blood pressure as well as increases in plasma cholesterol and homocysteine levels. ${ }^{8}$

This review indicated a lack of association between tea drinking and risk of cancers of oral cavity/pharynx and larynx. This may be attributed to the low consumption of tea, compared to caffeinated coffee, in European and American populations and the higher likelihood of misclassification. Findings from this review contradict those of Ren and colleagues, who found hot tea intake to be inversely associated with pharyngeal cancer. ${ }^{2}$ Furthermore, there are numerous reports on the cancer chemo-preventive activity of green tea, which contains powerful antioxidant polyphenols called catechins. Extracts of green tea and green tea polyphenols have exhibited inhibitory effects against the formation and development of tumours at different organ sites in animals, including the oral cavity and esophagus. ${ }^{9}$ Evidence on the protective effects of green tea against various cancers in human subjects tends to be weaker. Tsao et al. conducted a randomised placebocontrolled trial of green tea extract (GTE) in patients with high-risk oral premalignant lesions (OPL). Findings from the trial indicated that the OPL clinical response rate was higher in all GTE arms compared to placebo but did not reach statistical significance. ${ }^{10}$ A systematic review which included all prospective, controlled interventional studies and observational studies assessing the associations between green tea consumption and risk of cancer incidence or mortality, showed insufficient and conflicting evidence to give any firm recommendations regarding green tea consumption for cancer prevention. ${ }^{11}$

\section{Public health implications}

Coffee contains various compounds that have been reported to exert beneficial health effects due to their antioxidant and antineoplastic properties. The review by Galeone et al. provides a good evidence base to support an inverse association between caffeinated coffee drinking ( $>4$ cups per day) and risk of oral cavity and pharyngeal cancer. Although most studies have not reported an association between coffee consumption and coronary heart disease, these findings do not refute exclude that caffeinated coffee drinking could lead to adverse effects in certain patient groups, based on their genetic profile, health state or tolerance level. In addition, routine caffeine consumption may cause dependence, and abrupt discontinuation can produce irritability, mood shifts, headaches, drowsiness or fatigue. As for the association between tea intake and oral and pharyngeal cancers, more evidence is needed beyond animal models. This is especially important for green tea, which exhibits high cancer-preventative potential and appears to be well-tolerated and safe when consumed within the daily recommended allowance.

Imad Al-Dakkak Centre for Evidence-based Dentistry, Oxford, UK

1. Naganuma T, Kuriyama S, Kakizaki M, et al. Coffee consumption and the risk of oral, pharyngeal and esophageal cancers in Japan: the Miyagi Cohort Study. Am J Epidemiol 2008; 168: 1425-1432

2. Ren JS, Freedman ND, Kamangar F, et al. Tea, coffee, carbonated soft drinks and upper gastrointestinal tract cancer risk in a large United States prospective cohort study. Eur J Cancer 2010; 46: 1873-1881.

3. Je Y, Liu W, Giovannucci E. Coffee consumption and risk of colorectal cancer: a systematic review and meta-analysis of prospective cohort studies. Int J Cancer 2009; 124: $1662-1668$.

4. Galeone C, Turati F, La Vecchia C, et al. Coffee consumption and risk of colorectal cancer: a meta-analysis of case-control studies. Cancer Causes Control 2010; 21: 1949-1959.

5. Bravi F, Scotti L, Bosetti $C$, et al. Coffee drinking and endometrial cancer risk: a meta-analysis of observational studies. Am J Obstet Gynecol 2009; 200: 130-135.

6. Sugiyama K, Kuriyama S, Akhter M, et al. Coffee consumption and mortality due to all causes, cardiovascular disease and cancer in Japanese women. J Nutr 2010; 140: 1007-1013.

7. Giggey $\mathrm{PP}$, Wendell $\mathrm{CR}$, Zonderman $\mathrm{AB}$, et al. Greater coffee intake in men is associated with steeper age-related increases in blood pressure. Am J Hypertens 2011; 24: 310-315.

8. Riksen NP, Smits P, Rongen GA. The cardiovascular effects of methylxanthines. Handb Exp Pharmacol 2011; 200: 413-437.

9. Yang CS, Wang X. Green tea and cancer prevention. Nutr Cancer 2010; 62: 931-937.

10. Tsao AS, Liu D, Martin J, et al. Phase II randomised, placebo-controlled trial of green tea extract in patients with high-risk oral premalignant lesions. Cancer Prev Res 2009; 2: $931-941$.

11. Boehm K, Borrelli F, Ernst E, et al. Green tea (Camellia sinensis) for the prevention of cancer. Cochrane Database Syst Rev. 2009; (3): CD005004.

Evidence-Based Dentistry (2011) 12, 23-24. doi:10.1038/sj.ebd.6400780 